\title{
Conducting comprehensive scoping reviews to systematically capture the landscape of a subject matter
}

\author{
Mashrur R. Kazi ${ }^{1}$, Nashit Chowdhury ${ }^{1,2}$, Mohammad Chowdhury ${ }^{1}$, Tanvir C. Turin ${ }^{1,2}$
}

\section{AFFILIATION}

1 Department of Family Medicine, Cumming School of Medicine, University of Calgary, Calgary, Canada

2 Department of Community Health Sciences, University of Calgary, Calgary, Canada

\section{CORRESPONDENCE TO}

Tanvir Turin. Department of Family Medicine, Cumming School of Medicine, University of Calgary, Calgary, Alberta, Canada. E-mail: turin. chowdhury@ucalgary.ca ORCID ID: https://orcid.org/0000-0002-74995050

\section{KEYWORDS}

scoping review, comprehensive search, review typology, research methods, research team, manuscript components

Received: 6 October 2021, Revised: 7 November 2021,

Accepted: 9 November 2021

\begin{abstract}
Scientific publications or health research publications have seen a dramatic increase during the last few decades. Given this rapid production of information, literature reviews have become an imperative cornerstone for researchers to gain a better understanding of the research landscape that they are investigating. One type of literature review that has shown great utility is the scoping review, with its great efficiency in mapping existing literature and synthesizing
\end{abstract}

knowledge. A step-by-step guide is provided in this article as an introduction to those who are interested in conducting scoping reviews in order to acquire a better picture of the research landscape. To help better understand the intricacies to conducting a scoping review, each step of the process is clearly explained and elaborated on. Each step is also followed by examples from published literature. The intended audience includes students and researchers new to scoping reviews.

\section{INTRODUCTION}

$\overline{\text { Scientific publications or health research publications have }}$ seen a dramatic increase during the last few decades. The US National Institute of Health reported that the number of publications indexed by MEDLINE at the National Library of Medicine was 712,675 in 2009 and this number increased to 956,390 in 2019 . There was a $3.4 \%$ increase in publications indexed per year across the decade ${ }^{1}$. In this era of digitalization of information, the concept of grey literature as a body of information has surfaced in recent years due to the explosion of literature dissemination through nonconventional channels ${ }^{2}$. Grey literature databases are now also recognized as supplementary to academic databases ${ }^{2}$.

To conduct research on a topic of interest, it is imperative to have a clear understanding of what has been done so far on the chosen topic. A comprehensive understanding of the work done so far reduces the possibility of duplication and helps researchers to plan and conduct research to advance our understanding of the topic. Thus, good homework is crucial before starting research on the chosen topic. A goal of good homework can be achieved by conducting a literature review. Generally, any well-done reviews would be undertaken through the phases shown in Figure 1.

A literature review can range from a 'simple narrative write-up' to 'comprehensive in-depth information extraction and summarization'. Nowadays more and more authors are conducting reviews for their research purpose. Some are doing them to gain an understanding of their research landscape, some are doing them to summarize what has been done so far, or some are coming up with pooled estimates to quantify the relationship between two variables. In this context, capturing the variability in objectives and related depth in methodology, several types of literature review have been used by the researchers ${ }^{3,4}$.

A type of literature review that has shown great optimization and utility is the scoping review ${ }^{5,6}$. Scoping reviews are efficient at mapping existing literature on a given topic and synthesizing this knowledge. The nature of scoping reviews allows the researchers to capture the breadth of a research topic and are particularly useful in identifying the 
Figure 1. General steps of review

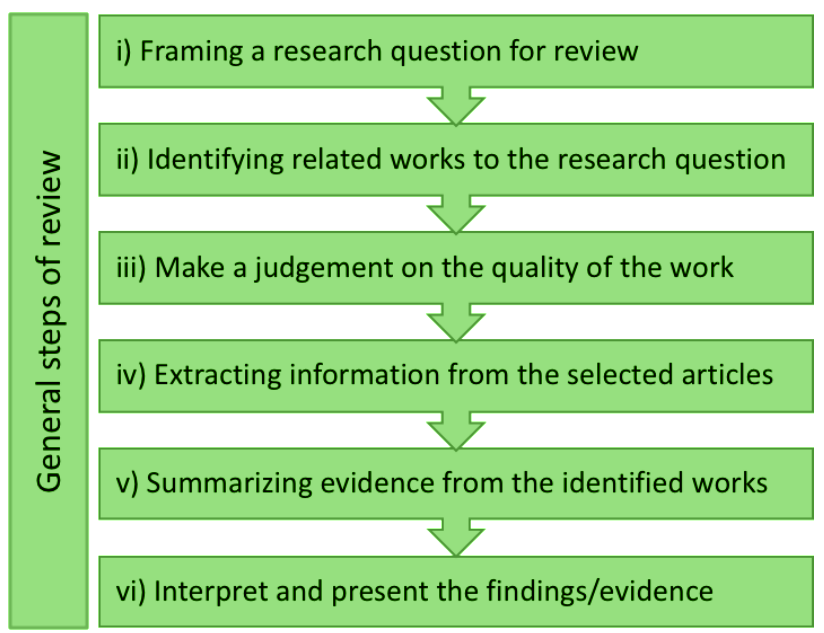

research landscape of a topic. Scoping reviews also provide a great utility in identifying research gaps in the literature and potential future steps in research. While this form of review is often used to identify the potential scope of a specific systematic review, it can also be used to summarize and disseminate information regarding research findings in the form of publications. Table 1 summarizes the differences between scoping and systematic reviews.

\section{METHODOLOGICAL APPROACH}

In this article, we will present in detail how to conduct a systematic scoping review based on common methodological frameworks ${ }^{5,7}$. These steps are described below with the example of a recently published review from our research group ${ }^{8}$. This review was conducted to summarize the research conducted on injury for Canadian immigrants ${ }^{8}$. Figure 2 outlines the steps for conducting a scoping review.

\section{Step 1: Narrowing down on a research question}

The first step in conducting a scoping review is to develop a research question based on the objectives and specific inquiries of the review. The purpose/objective of the scoping review is then used to guide the development of the inclusion and exclusion criteria. The principal idea or focus should be driven throughout the article to identify the breadth and depth of the scoping review.

The PICOS framework can be used to define the breadth and depth of the research question ${ }^{9}$. PICOS is an acronym for: Population, Intervention, Comparison, Outcome, and Study design; and is often used for quantitative synthesis. Using these four parameters, a researcher can determine the breadth and depth of their research question. It is a fundamental part of the process as it can help to develop the research question and shape the focus of the scoping review. Alongside the PICOS framework, there are other

frameworks that the researchers can use depending on the objective of the review. These include: Population, Intervention, Comparator(s), Outcomes, Context (PICOC); Condition, Context, Population (CoCoPop); Population, Index Test, Reference Test, Diagnosis of Interest (PIRD); Population, Exposure, Outcome (PEO); Population, Prognostic Factors, Outcomes (PFO); and Studies, Data, Methods, Outcomes $(\mathrm{SDMO})^{10}$. An example of the PICOS framework is provided in Table 2.

\section{Step 2: Creating exclusion/inclusion criteria}

Having well-defined inclusion and exclusion criteria are crucial to the process of pursuing appropriate literature for a scoping review ${ }^{11}$. The inclusion and exclusion criteria are a reflection of the scope of the article and a guide that

\section{Table 1. Differences between scoping review and systematic review}

\section{Scoping review}

Summarizing the different types of research on a topic of interest

Data charted according to selected key themes

Broad capture of information

Identify and examine the methodology of research being studied

Optional bias assessment Identify research gaps

\section{Systematic review}

Concentrating specific research evidence on a focused topic of interest Defined protocol in extracting data (quantitative or qualitative)

Focused capture of information

Investigate and examine various results and conflicts

Mandatory bias assessment Present cumulative evidence 


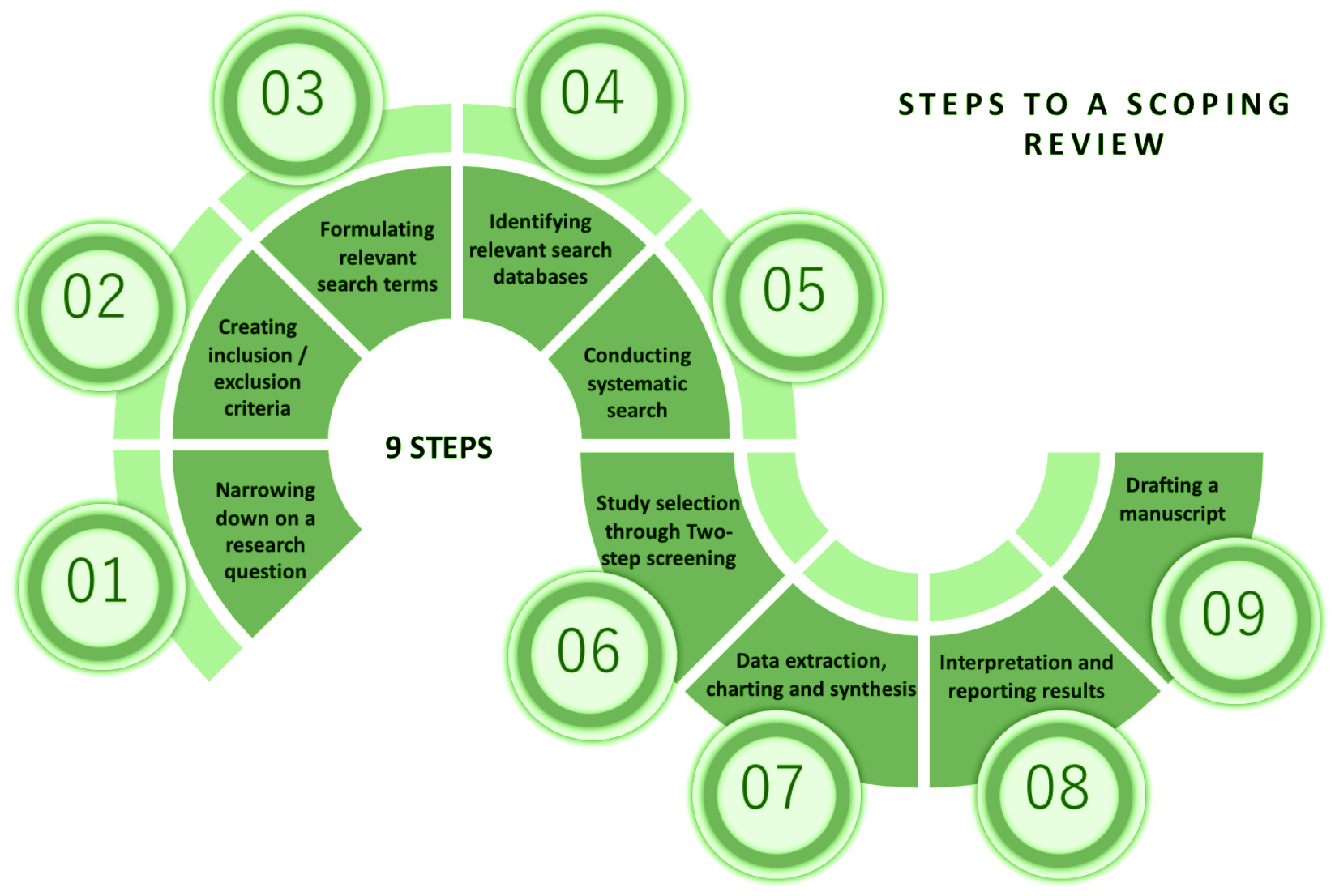

determines the specificity or comprehensiveness of the review $^{12}$. It acts as a guide for the reviewers to decide on what to include in the review and what to exclude. The reasoning and rationale behind each established criterion must be stated and justified clearly by the reviewers, so that there is no room for confusion to the reader. The languages and timeline considered for the inclusion criteria must be explicitly stated here by the reviewers (Table 3 ).

\section{Step 3: Formulating relevant keywords}

Formulating relevant keywords is a fundamental step required in the process of synthesizing a scoping review ${ }^{13}$. To determine relevant keywords, an initial limited search must be performed on the topic. This search then allows the researchers to screen the title and abstract of other articles and to identify relevant terms used by these articles. This initial limited search allows the researchers to further define their scope of the review and allows them to be more specific with their keywords so that the most relevant articles are found in the search ${ }^{14}$. This search also lets the researchers identify relevant search databases (Step 4) that need to be searched for this scoping review. This initial search allows to redefine the search strategy. Furthermore, search terms can be generally divided into two categories: Medical Subject Heading (MeSH), and keywords. MeSH terms are defined as terminology that is used by the National Library of Medicine database to catalogue and index health-related information ${ }^{15}$. These specific terms are used to index existing

Table 2. Elaboration and explanation of PICOS with an example of a systematic review

\section{Elaboration of PICOS}
P: Population
I: Intervention
C: Comparison
0: Outcome

S: Study Design

Example: Virtual Reality in Stroke Rehabilitation: A Systematic Review of its Effectiveness for Upper Limb Motor Recovery (PMID: 17517575)

P: Patients with post-stroke hemiplegia

I: Immersive or non-immersive virtual reality

C: Conventional therapy or no therapy

0: Differences between groups

S: Experimental studies including randomized controlled trials (RCTs) 
literature and therefore can provide relevant articles when identified as a keyword in a systematic search. Additionally, keywords are words or phrases that can be used to search a database ${ }^{16}$. Keywords become extremely useful when there are no previous MeSH terms determined for a specific topic. When conducting a scoping review, it is important to break down the PICOS, the research question, and the objectives into smaller components, such that relevant keywords for each section can be formulated and later combined for the systematic search (Table 4). This allows for an extensive search of the existing literature, which is necessary for a scoping review.

\section{Step 4: Identifying relevant search databases}

After formulating and finalizing relevant key terms necessary for the search, relevant search databases must be identified $^{2,13}$. It is important that the researchers identify the most relevant databases to search as these are often specific to the topic being researched. As mentioned in Step 3, an initial limited search provides the researchers with an idea of relevant search databases. Similar to the keywords, relevant databases to search for the scoping review are derived from the principal research question. It is recommended to search as many relevant article databases as possible in order to properly address the research question (Table 5). It is also important to recognize that this process will yield many duplicates as well, which can be later removed by using the appropriate software.

Alongside academic databases, grey literature databases have become a fundamental part of scoping reviews ${ }^{2}$. Grey literature is non-conventional and rapid sources of information that allow accessing various types of documents that are produced by governments, organizations, business institutions, etc., and not published by professional publishers ${ }^{2}$. Generally, grey literature is not peer-reviewed. Search engines such as Google and Google Scholar have become a cornerstone for grey literature searches, as many

\section{Table 3. Inclusion and exclusion criteria used in the example scoping review article $^{8}$}

$\begin{array}{ll}\begin{array}{l}\text { Inclusion Criteria } \\ \begin{array}{l}\text { Include studies only on } \\ \text { immigrants }\end{array}\end{array} & \begin{array}{l}\text { Exclusion Criteria } \\ \text { Exclude books, reviews, } \\ \text { letters, commentaries, or } \\ \text { editorials }\end{array} \\ \begin{array}{l}\text { Include studies only } \\ \text { published in English }\end{array} & \begin{array}{l}\text { Exclude studies published in } \\ \text { languages other than English }\end{array} \\ \begin{array}{l}\text { Include original research } \\ \text { studies only }\end{array} & \begin{array}{l}\text { Exclude studies on the } \\ \text { population who are not } \\ \text { Canadian immigrants }\end{array} \\ \begin{array}{l}\text { Include studies conducted in } \\ \text { Canada }\end{array} & \\ \begin{array}{l}\text { Include studies on any aspect } \\ \text { of injury }\end{array} & \end{array}$

websites are powered by this search engine ${ }^{17}$. Grey literature databases are similar to academic databases, as specific ones are needed to be geared to the research question.

\section{Step 5: Conducting a systematic search}

Once finalized, the relevant keywords and databases (both academic and grey) must be used to create a search strategy. The search strategy should be comprehensive so

\section{Table 4. Search terms developed for the example scoping review article ${ }^{8}$}

\section{Search-terms/keywords for immigrant}

immigrant* [Keyword]; emigrant* [Keyword]; emigrants and immigrants [MeSH]; 'undocumented immigrant*' [Keyword] a; 'undocumented emigrant*' [Keyword]; undocumented immigrants [MeSH]; alien* [Keyword]; foreigner* [Keyword]; newcomer* [Keyword]; refugee* [Keyword]; refugees [MeSH]

\section{Search-terms/keywords for injury}

injur* [Keyword]; wounds and injuries [MeSH]; wound* [Keyword]; accident* [Keyword]; 'accident proneness' [Keyword, MeSH]; 'traffic accident*'[Keyword]; accidents, traffic [MeSH]; 'motor vehicle crash' [Keyword]; 'occupation* injur*' [Keyword]; occupational injuries [MeSH]; accidents, occupational [MeSH]; 'occupational accident*' [Keyword]; 'risk factor*' [Keyword]; risk factors [MeSH]; disabilit* [Keyword]; 'disabled person*' [Keyword]; disabled persons [MeSH]; 'injury prevention' [Keyword]; 'accident prevention' [Keyword, MeSH]

Using '*', '\$', or ':' at the end in Ovid Medline and some other database search engines allows to include unlimited suffix variations including alternative spelling, plurals, and a different form of parts of speech. For example, searching using 'injur*' keyword will retrieve all results having 'injury', 'injuries', and 'injured' keywords.

Table 5. Search databases used for the example scoping review article

Academic databases

for published articles

MEDLINE (Ovid)

Grey literature databases

EMBASE

Google Scholar

EBM Reviews

ProQuest (theses and dissertations)

OpenDOAR (institutional

PubMed repositories)

CINAHL Health Sciences Online (HSO)

(TRIP)

MEDLINE (Ebsco) OAISTER (WorldCat)

Web of Science Canadian Institute for Health Information (CIHR)

Public Health Agency of Canada (PHAC)

Health Canada

National Institutes of Health (NIH) 
that the search itself is relevant to the research question and extensive. In different databases, different search strategies are employed. In general, search terms within each component of the research question (as guided by PICOS) are

Table 6. MEDLINE search strategy used for the example scoping review article

\section{MEDLINE search strategy}

1. 'immigrant'.ab,ti.

2. 'emigrant*'.ab,ti.

3. exp 'Emigrants and Immigrants'/

4. 'undocumented immigrant*'ab,ti.

5. 'undocumented emigrant*'.ab,ti.

6. exp Undocumented Immigrants/

7. 'alien*'.ab,ti.

8. 'foreigner*'.ab,ti.

9. 'newcomer*'.ab,ti.

10. 'refugee*'.ab,ti.

11. exp Refugees/

12. 1 or 2 or 3 or 4 or 5 or 6 or 7 or 8 or 9 or 10 or 11

13. 'injur*'.ab,ti.

14. exp 'Wounds and Injuries' /

15. 'wound"'.ab,ti.

16. 'accident*'.ab,ti.

17. 'accident proneness'.ab,ti.

18. exp Accident Proneness/

19. 'traffic accident* 'ab,ti.

20. exp Accidents, Traffic/

21. 'motor vehicle crash'.ab,ti.

22. 'occupation* injur* 'ab,ti.

23. exp Occupational Injuries/

24. exp Accidents, Occupational/

25. 'occupational accident* 'ab,ti.

26. 'risk factor*'.ab,ti.

27. exp Risk Factors/

28. 'disabilit*'.ab,ti.

29. 'disabled person* 'ab,ti.

30. exp Disabled Persons/

31. 'injury prevention'ab,ti.

32. 'accident prevention'.ab,ti.

33. exp Accident Prevention/

34. 13 or 14 or 15 or 16 or 17 or 18 or 19 or 20 or 21 or 22 or 23 or 24 or 25 or 26 or 27 or 28 or 29 or 30 or 31 or 32 or 33

35. 12 and 34 first combined using the Boolean operator 'OR' to produce search results that include a wide selection. Next, the search results produced from the last step are combined using the Boolean operator 'AND' to conduct a complete systematic search that encompasses all parts of the research question. Table 6 provides an example of carrying out a search by utilizing search terms in MEDLINE, which is one of the most popular and comprehensive databases in medical research.

Given the fast-paced nature of grey literature, the search strategy regarding this domain focuses mainly on keyword searching. Researchers employ universal strategies such as using Boolean operators ('AND' and 'OR') to combine search terms. Grey literature databases such as Google scholar are then searched using these terms. Furthermore, due to the algorithm that determines the relevance of the results yielded from the search, only the first 100 results or the first 10 pages are taken into consideration for the scoping review screening ${ }^{2}$.

\section{Step 6: Study selection through two-step screening}

Relevant articles are identified through a two-step screening process that allows the researchers to screen out irrelevant literature, as systematic searches can often yield a high number of articles. The first step of studyselection includes screening the title and abstract of the articles that were found through the systematic search. Two independent reviewers start this process at the same time. The involvement of two independent reviewers ensures the rigor of inclusion/exclusion by minimizing the potential influence of individual-level bias. Generally, the agreements/ disagreements between the two reviewers are estimated through Cohen's kappa coefficient statistics which is used to measure the inter-rater reliability ${ }^{18}$. It is important that reviewers have a high percentage of agreement in screening decisions. It is a good idea to conduct a pilot screening of 50-100 studies by both reviewers and cross-check the decisions. Any disagreements need to be discussed within the team to identify the reasons for the disagreements, which will help the two reviewers to have a common understanding of the application of inclusion/exclusion mechanisms and screening processes. If the title and abstract meet the inclusion criterion set by the researchers, then the screening can move on to the second stage of the study selection which is full-text screening ${ }^{19}$. If there are doubts at this stage about a particular article based on the title and abstract, the researchers must include the study in the next stage of fulltext screening. At this stage, the researchers need to read the full article to see if it should be included in the scoping review. If there are disagreements, the reviewers must come to a consensus to either accept or reject the article based on the inclusion criteria. The final decision to include a study is done at this stage. Researchers also manually go through the reference lists or bibliography of the final selected articles to identify if any relevant others have been missed. This technique is known as Snowballing, Citation Mining or Pearl 
Figure 3. Literature search and selection schematic using flow diagram used for the example scoping review $\operatorname{article~}^{8}$

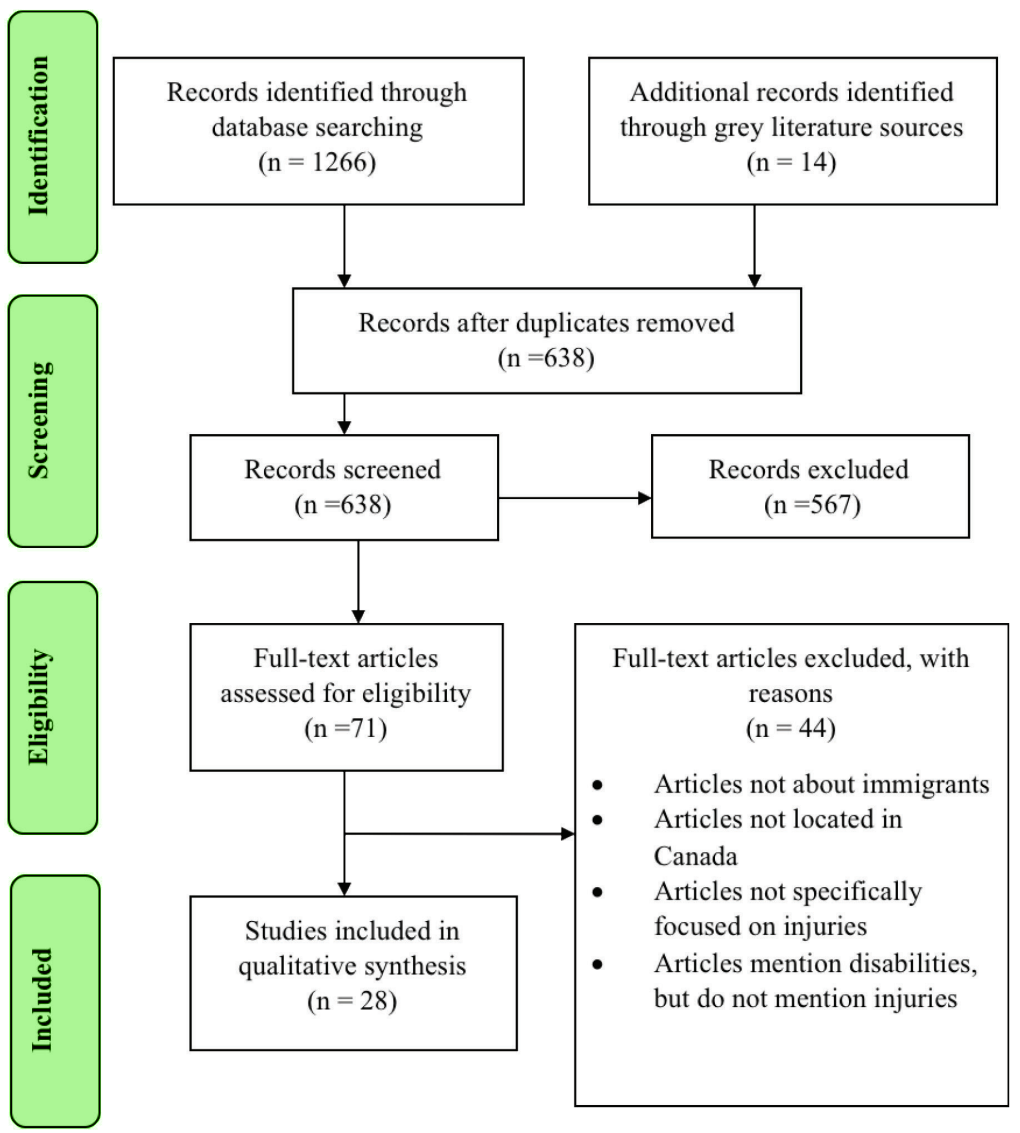

Growing $^{20}$. Software such as MS Excel ${ }^{21}$ or Covidence ${ }^{22}$ as well as referencing software like EndNote ${ }^{23}$ or RefWorks ${ }^{24}$ are often used to aid this screening. In scoping reviews, all these steps are summarized using a PRISMA flow diagram ${ }^{25,26}$. The flow diagram maps out the number of records identified, included and excluded, and the reasons for exclusion. Figure 3 shows the search and screening flow diagram used in the example scoping review article. It depicts the flow of information through the different phases of the scoping review; the number of articles identified from the search, the number remaining after removal of duplicates, the number excluded through the two-step screening, and the number of studies finally selected for synthesis.

\section{Step 7: Data extraction, charting and synthesis}

In scoping reviews, once the studies have been selected, the key information presented in those studies needs to be extracted and charted. There are different ways through which information can be presented in a scoping review. Information from the results may be presented in tables, charts, and diagrams, in a logical way so that the reader can understand the key information. The information being charted must reflect the research question and the principal inquiry of the scoping review. The first table normally presents the basic information regarding the studies included in the scoping review: year of publication, demographic information, author, country of research, type of research, etc. The subsequent tables or charts are dependent on the researchers preference on how to express the findings in a logical and easy to follow manner.

\section{Step 8: Interpretation and reporting results}

All the information extracted from the articles needs to be summarized and collated in a logical manner that addresses the research question of the scoping review. The summary of information should clearly describe the purpose and results of each study included in the scoping review. As information has been charted and extracted, it is now the responsibility of the researchers to thematically categorize the information in a way that is aligned with the research aim of the scoping review. Categorization of the information depends on the results of the literature included in the scoping review. Furthermore, especially for scoping reviews, it is important that gaps in the research are identified and presented to the 
Figure 4. Components of a scoping review manuscript

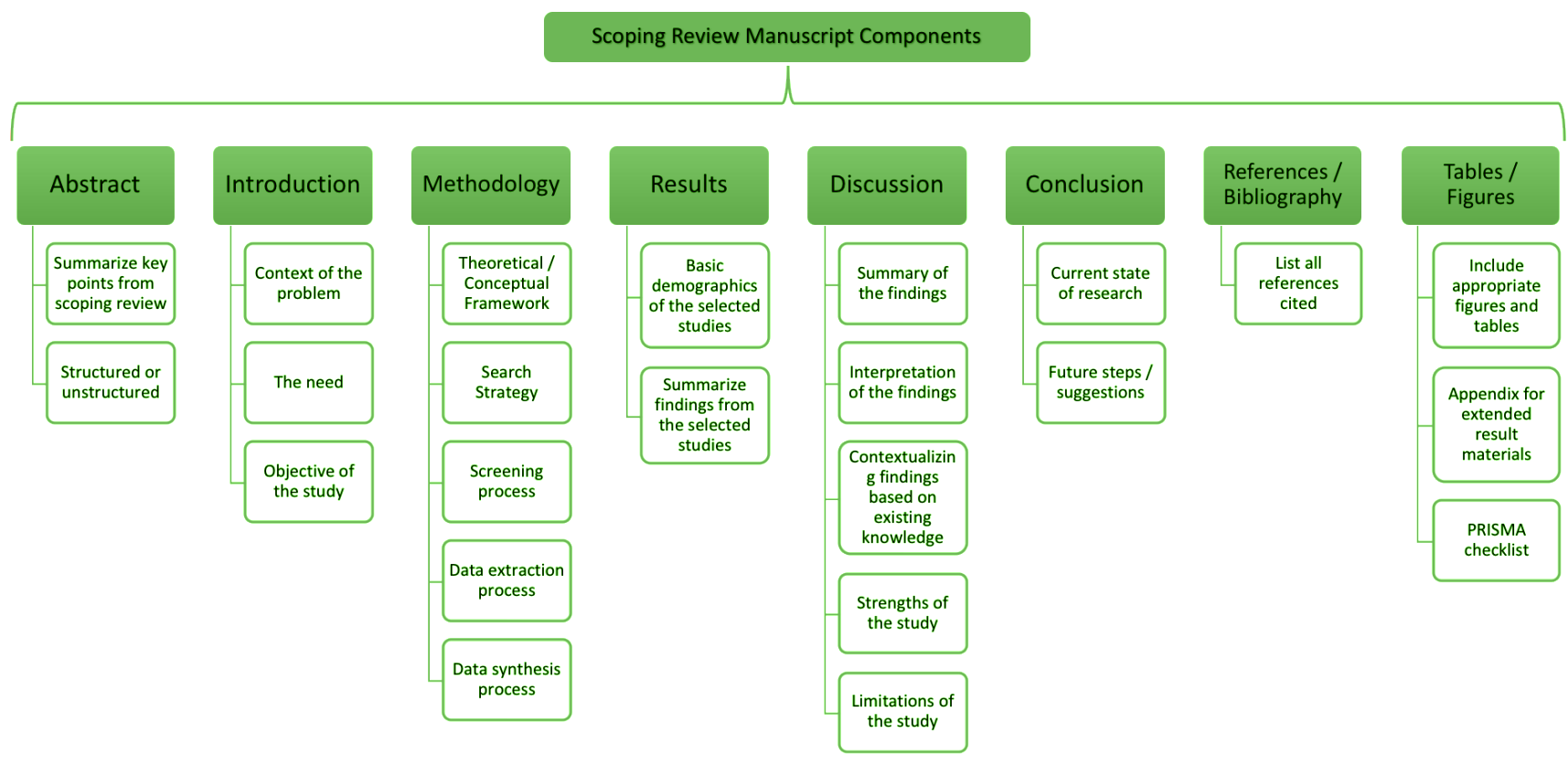

readers to give them an understanding of the landscape of what has been done and what future research needs to be undertaken.

\section{Step 9: Drafting a manuscript}

The final step of the process of conducting a scoping review is to draft a manuscript for submission to a journal. A scoping review manuscript generally consists of the sections shown in Figure 4.

\section{PRISMA checklist for scoping review}

A checklist, which is known as Preferred Reporting Items for Systematic reviews and Meta-Analyses (PRISMA), has been developed to make sure that a minimum set of items are ensured by researchers to improve the reporting of systematic reviews and meta-analyses ${ }^{26}$. In 2018, a PRISMA extension for scoping reviews was published, which is known as PRISMA-ScR Checklist ${ }^{27}$. Nowadays, many journals require the researchers to complete a PRISMA-ScR checklist when they submit their scoping reviews for publication. This checklist has 22 items listed across all the components of a scoping review manuscript. Details on the PRISMA-ScR can be found on the following site: http://www.prismastatement.org/Extensions/ScopingReviews.

\section{Study quality evaluations for scoping reviews}

Though it is not yet that common or mandatory to evaluate the selected studies in a scoping review for quality assessment, researchers can conduct a critical appraisal of the selected studies in their scoping reviews. A set of criteria need to be determined to evaluate a study's (included for the final synthesis in scoping review) quality. In general, this set of criteria is proposed based on different metrics of the articles that can be compared, such as research purpose, research question, and methodological approaches. There are some formal checklists available that are suggested to be used for assessing the quality of a study. Separate quality assessment tools are used for different types of studies (quantitative or qualitative), or different types based on analytical methodologies used in the studies ${ }^{28}$. A detailed description of the basics of study quality assessment has been reported elsewhere ${ }^{28}$.

\section{Creating a scoping review team}

In order to conduct a successful scoping review, a review team based on different skills needs to be assembled. These skills are based on different activities that need to be undertaken to conduct a scoping review. At least two reviewers with expertise in the methodological and content directions are needed in a scoping review team. The team can be expanded with an experienced librarian who can undertake the comprehensive search process. The team is responsible for determining the comprehensiveness and breadth of the scoping review. Potential limitations must be addressed by the team as well. Figure 5 outlines the major skills needed to be ensured in the composition of a scoping review team.

\section{CONCLUSIONS}

Scoping reviews can be a very useful tool in the context of summarizing and disseminating knowledge on the landscape of a topic of research. The nature of scoping reviews allows 
Figure 5. The composition of a review team

\begin{tabular}{|c|c|c|c|c|}
\hline \multicolumn{5}{|c|}{ Composition and Roles of Review Team } \\
\hline $\begin{array}{l}\text { Content } \\
\text { Expert }\end{array}$ & $\begin{array}{l}\text { Search } \\
\text { Expert }\end{array}$ & $\begin{array}{c}\text { Two } \\
\text { Independent } \\
\text { Reviewers }\end{array}$ & $\begin{array}{c}\text { Analysis } \\
\text { Expert }\end{array}$ & $\begin{array}{c}\text { Content } \\
\text { Expert }\end{array}$ \\
\hline $\begin{array}{l}\text { Designing the } \\
\text { Review }\end{array}$ & $\begin{array}{l}\text { Search Planning } \\
\text { \& Conduction }\end{array}$ & $\begin{array}{l}\text { Screening \& } \\
\text { Extraction }\end{array}$ & $\begin{array}{l}\text { Analysis \& } \\
\text { Synthesis }\end{array}$ & $\begin{array}{l}\text { Knowledge } \\
\text { Product }\end{array}$ \\
\hline $\begin{array}{l}\text { Prepares the } \\
\text { context of the } \\
\text { review } \\
\text { - Responsible } \\
\text { for developing } \\
\text { the research } \\
\text { question }\end{array}$ & $\begin{array}{l}\text { Develops } \\
\text { appropriate } \\
\text { search terms } \\
\text { Choosing } \\
\text { appropriate } \\
\text { databases } \\
\text { Conducting } \\
\text { the search } \\
\text { Compiling the } \\
\text { search results }\end{array}$ & $\begin{array}{l}\text { Conduct two- } \\
\text { step screening } \\
\text { (title-abstract } \\
\text { and full-text) } \\
\text { Data extraction } \\
\text { from the final } \\
\text { selected } \\
\text { studies } \\
\text { Quality } \\
\text { assessment of } \\
\text { final selected } \\
\text { studies }\end{array}$ & $\begin{array}{l}\text { - Analyze the } \\
\text { extracted } \\
\text { data } \\
\text { - Summarizing } \\
\text { the findings }\end{array}$ & $\begin{array}{l}\text { - Interpret } \\
\text { findings } \\
\text { - Present } \\
\text { findings as a } \\
\text { manuscript } \\
\text { - Create other } \\
\text { knowledge } \\
\text { products as } \\
\text { required }\end{array}$ \\
\hline
\end{tabular}

researchers to quickly and efficiently identify research gaps and suggests the next steps in the specific field of research.

\section{REFERENCES}

1. Citations Added to MEDLINE® by Fiscal Year. National Library of Medicine. Updated November 3, 2020. Accessed November 7, 2021. https://www.nlm.nih.gov/bsd/stats/ cit_added.html

2. Vaska M, Chowdhury M, Naidu J. Exploring all that is Grey in the Health Sciences: What is Grey Literature and How to use it for Comprehensive Knowledge Synthesis. Journal of National Heart Foundation of Bangladesh. 2019;8(1):1419. Accessed November 7, 2021. https://www.nhf.org.bd/ Journal/Web_Upload/JNHFB_2019/2019_JNHFB_Vol\%20 8\%20Issue $\% 201 / 4 \% 20$ Exploring\%20all\%20that $\% 20$ is $\% 20$ Grey\%20in\%20the\%20Health\%20Sciences.pdf

3. Ahmed S, Vaska M, Turin TC. Conducting a Literature Review in Health Research: Basics of the Approach, Typology and Methodology. Journal of National Heart Foundation of Bangladesh. 2016;5(2):44-51. Accessed November 7, 2021. https://acfp.ca/wp-content/uploads/2018/04/101-Typesof-Review-Salim_Marcus_Turin.pdf

4. Samnani SS, Vaska M, Ahmed S, Turin TC. Review Typology: The Basic Types of Reviews for Synthesizing Evidence for the Purpose of Knowledge Translation. J Coll Physicians Surg Pak. 2017;27(10):635-641. Accessed November 7, 2021. https://jcpsp.pk/archive/2017/Oct2017/10.pdf

5. Peters MDJ, Godfrey CM, Khalil H, McInerney P, Parker D, Soares CB. Guidance for conducting systematic scoping reviews. Int J Evid Based Healthc. 2015;13(3):141-146.
doi:10.1097/XEB.0000000000000050

6. Grant MJ, Booth A. A typology of reviews: an analysis of 14 review types and associated methodologies. Health Info Libr J. 2009;26(2):91-108. doi:10.1111/j.1471-1842.2009.00848.x

7. Arksey H, O’Malley L. Scoping studies: towards a methodological framework. Int J Soc Res Methodol. 2005;8(1):19-32. doi:10.1080/1364557032000119616

8. Kazi MR, Ferdous M, Rumana N, Vaska M, Turin TC. Injury among the immigrant population in Canada: exploring the research landscape through a systematic scoping review. Int Health. 2019;11(3):203-214. doi:10.1093/inthealth/ihy086

9. Schardt C, Adams MB, Owens T, Keitz S, Fontelo P. Utilization of the PICO framework to improve searching PubMed for clinical questions. BMC Med Inform Decis Mak. 2007;7(1):16. doi:10.1186/1472-6947-7-16

10. Munn Z, Stern C, Aromataris E, Lockwood C, Jordan Z. What kind of systematic review should I conduct? A proposed typology and guidance for systematic reviewers in the medical and health sciences. BMC Med Res Methodol. 2018;18(1):5. doi:10.1186/s12874-017-0468-4

11. Meline T. Selecting Studies for Systematic Review: Inclusion and Exclusion Criteria. Contemp Issues Commun Sci Disord. 2006;33(Spring):21-27.doi:10.1044/cicsd_33_S_21

12. Carnwell R, Daly W. Strategies for the construction of a critical review of the literature. Nurse Educ Pract. 2001;1(2):57-63. doi:10.1054/nepr.2001.0008

13. Ahmed S, Vaska M, Turin T. Comprehensive Systematic Search Process of Health Literature: Hunting Pearls out of the Sea. Journal of National Heart Foundation of Bangladesh. 2016;5(1):12-16. Accessed November 7, 2021. https://www. 
nhf.org.bd/Journal/Web_Upload/JNHFB_2016/2016_JNHFB_ Vol\%205\%20Issue\%201/12.\%20CSSPHL.pdf

14. Aromataris E, Riitano D. Constructing a Search Strategy and Searching for Evidence: A guide to the literature search for a systematic review. Am J Nurs. 2014;114(5):49-56. doi:10.1097/01.NAJ.0000446779.99522.f6

15. Introduction: What is MeSH? National Library of Medicine. Accessed November 7, 2021. https://www.nlm.nih.gov/bsd/ disted/meshtutorial/introduction/index.html

16. Literature searching explained: Develop a search strategy. University of Leeds Library. Accessed November 7, 2021. https://library.leeds.ac.uk/info/1404/literature_ searching/14/literature_searching_explained/4

17. Haddaway NR, Collins AM, Coughlin D, Kirk S. The Role of Google Scholar in Evidence Reviews and Its Applicability to Grey Literature Searching. PLoS One. 2015;10(9):e0138237. doi:10.1371/journal.pone.0138237

18. Kraemer HC. Kappa Coefficient. In: Balakrishnan N, Colton T, Everitt B, Piegorsch W, Ruggeri F, Teugels J, eds. Wiley StatsRef: Statistics Reference Online. Wiley; 2014:1-4.

19. Lefebvre C, Glanville J, Briscoe S, et al. Searching for and selecting studies. In: Higgins JTP, Thomas J, Chandler J, et al, eds. Cochrane Handbook for Systematic Reviews of Interventions. 2nd ed. John Wiley \& Sons; 2019:67-107.

20. Web of Science: Home. Icahn School of Medicine at Mount Sinai. Accessed November 7, 2021. https://libguides.mssm. edu/webofscience

21. Microsoft Excel. Microsoft. Accessed November 7, 2021. https://www.microsoft.com/en-us/microsoft-365/excel

22. Covidence. Accessed November 7, 2021. https://www. covidence.org/

23. EndNote. Clarivate Analytics. Accessed November 7, 2021. https://endnote.com/

24. RefWorks. ProQuest LLC. Accessed November 7, 2021. https://refworks.proquest.com/

25. Liberati A, Altman DG, Tetzlaff J, et al. The PRISMA statement for reporting systematic reviews and meta-analyses of studies that evaluate health care interventions: explanation and elaboration. J Clin Epidemiol. 2009;62(10):e1-e34. doi:10.1016/j.jclinepi.2009.06.006

26. Moher D, Liberati A, Tetzlaff J, Altman DG; PRISMA Group. Preferred Reporting Items for Systematic Reviews and Meta-Analyses: The PRISMA Statement. PLoS Med. 2009;6(7):e1000097. doi:10.1371/journal.pmed.1000097

27. Tricco AC, Lillie E, Zarin W, et al. PRISMA Extension for Scoping Reviews (PRISMA-ScR): Checklist and Explanation. Ann Intern

CONFLICTS OF INTEREST

The authors have completed and submitted the ICMJE Form for Disclosure of Potential Conflicts of Interest and none was reported.

FUNDING

There was no source of funding for this research.
Med. 2018;169(7):467-473. doi:10.7326/M18-0850

28. MZI C, Turin TC. Synthesizing Quantitative and Qualitative Studies in Systematic Reviews: The Basics of Meta-analysis and Meta-synthesis. Journal of National Heart Foundation of Bangladesh. 2019;8(2):55-61. Accessed November 7, 2021. https://www.nhf.org.bd/Journal/Web_Upload/ JNHFB_2019/2019_JNHFB_Vol\%208\%20Issue\%202/4\%20 Synthesizing\%20Quantitative\%20and\%20Qualitative\%20 Studies.pdf
DATA AVAILABILITY

Data sharing is not applicable to this article as no new data were created.

PROVENANCE AND PEER REVIEW

Not commissioned; externally peer reviewed. 